\title{
Estrés percibido en estudiantes de enfermería durante el confinamiento obligatorio por Covid-19
}

Stress Perceived by Nursing Students during the Mandatory COVID-19 Lockdown

Estresse percebido em estudantes de enfermagem durante 0 confinamento obrigatório por Covid-19

Como citar este artículo:

Muvdi Muvdi Yolanda, Malvaceda Frías Eynick, Barreto Vásquez Marisol, Madero Zambrano Kendy, Mendoza Sánchez Xilene, Bohórquez Moreno Cristina. Estrés percibido en estudiantes de enfermería durante el confinamiento obligatorio por Covid-19. Revista Cuidarte. 2021;12(2):e1330. http://dx.doi.org/10.15649/cuidarte.1330

Revista Cuidarte

Rev Cuid. May - Ago 2021; 12(2): e1330

doi http://dx.doi.org/10.15649/cuidarte.1330

E-ISSN: 2346-3414

(1) Yolanda Muvdi Muvdi ${ }^{1}$

(1) Eynick Malvaceda Frías ${ }^{2}$

(1) Marisol Barreto Vásquez ${ }^{3}$

(1) Kendy Madero Zambrano 4

(1) Xilene Mendoza Sánchez ${ }^{5}$

(1) Cristina Bohórquez Moreno ${ }^{6}$

1 Corporación Universitaria Rafael Núñez- Barranquilla., Colombia E-mail: ymuvdim27@curnvirtual.edu.co

2 Corporación Universitaria Rafael Núñez- Barranquilla, Colombia. E-mail: emalvacedaf27@curnvirtual. edu.co

3 Corporación Universitaria Rafael Núñez- Barranquilla, Colombia, E-mail: marisol.barreto@curnvirtual. edu.co.

4 Corporación universitaria Rafael Núñez- Cartagena, Colombia, Autora Correspondencia. E-mail: kendy.madero@curnvirtual.edu.co.

5 Corporación Universitaria Rafael Núñez, Barranquilla, Colombia. E-mail: xilene.mendoza@curvirtual. edu.co.

6 Corporación Universitaria Rafael Núñez,Barranquilla, Colombia. E-mail: cristina.bohorquez@ curnvirtual.edu.co.

\section{Resumen}

Introducción: el estrés académico es una reacción que activa la parte fisiológica, emocional, cognitiva y conductual que sufren los estudiantes ante ciertos agentes estresores, como la pandemia mundial del Covid-19. Objetivo: determinar el nivel de estrés percibido en el periodo de confinamiento por Covid-19 en estudiantes de Enfermería de una universidad de la región caribe Colombiana Materiales y métodos: estudio de analítico, participaron 398 estudiantes de Enfermería de una Universidad de la costa Caribe Colombiana, se emplearon dos instrumentos, una encuesta sociodemográfica y la escala denominada Estrés percibido 10. Resultados: El promedio de estrés fue de 18,83 5,19 , mostrando un nivel leve de estrés, siendo más bajo en personas de mayor edad (30-34 y >=35 años), ( $P=0,00)$, las mujeres presentaban una leve disminución del estrés percibido en comparación con los hombres $(\mathrm{P}=0,04)$ y las personas que habitan en el área rural presentan niveles de estrés más elevados - Discusión: El presente estudio revela efectos de tres meses de confinamiento por COVID-19 en una población de jóvenes universitarios que interrumpieron su formación educativa de forma presencial a remota, la cual provocó estrés en la población sujeto de estudio. Conclusión: Se evidenció que los hombres con menos edad presentan mayores niveles de estrés en comparación con las mujeres y a medida que aumenta la edad estos niveles disminuyen. El habitar en áreas rurales puede convertirse en un elemento estresor para los estudiantes.

Palabras clave: Estrés Psicológico; Coronavirus; Estudiantes del Área de la Salud

Recibido: junio 23 de 2020

Aceptado: febrero 2 de 2021

Publicado: mayo 18 de 2021 $\square *$ Correspondencia Kendy Madero Zambrano E-mail: kendy.madero@curnvirtual.edu.co. 


\title{
Stress Perceived by Nursing Students during the Mandatory COVID-19 Lockdown
}

\begin{abstract}
Introduction: Academic stress is a physiological, emotional, cognitive and behavioral response of students to some stressors such as the global COVID-19 pandemic. Objective: To determine the level of stress perceived by nursing students at a Colombian Caribbean university during the mandatory COVID-19 lockdown. Materials and Methods: An analytical study was conducted with 398 nursing students at a Colombian Caribbean university, who completed two instruments, a sociodemographic questionnaire and the Perceived Stress Scale (PSS-10). Results: Average stress rate was 18.83 5.19, indicating a mild level of stress but being lower in older students (30-34 and $>=35$ years) $(P=0.00)$. Women had a slightly lower rate of perceived stress compared to men $(P=0.04)$. Students living in rural areas showed to have higher stress levels. Discussion: This study revealed the impact of three months of restrictions during the COVID-19 lockdown in a population of young university students who have interrupted their inperson educational training to online training, causing academic stress in the study population. Conclusion: Younger men showed higher levels of stress compared to women, decreasing as their age increases. Living in rural areas may become a stressor for students.
\end{abstract}

Key words: Stress, Psychological; Coronavirus; Students, Health Occupations

\section{Estresse percebido em estudantes de enfermagem durante 0 confinamento obrigatório por Covid-19}

\section{Resumo}

Introdução: o estresse acadêmico é uma reação que ativa a parte fisiológica, emocional, cognitiva e comportamental sofrido pelos alunos em face de certos agentes estressores, como a pandemia global da Covid-19. Objetivo: determinar o nível de estresse percebido no período de confinamento por Covid-19 em alunos de Enfermagem de uma universidade da região do Caribe colombiano Materiais e métodos: estudo analítico participaram 398 estudantes de Enfermagem de uma Universidade da costa do Caribe Colombiana, foram utilizados dois instrumentos, um levantamento sociodemográfico e a escala denominada Estresse percebido 10. Resultados: A média de estresse foi de 18,83 $\pm 5,19$, mostrando um nível leve de estresse, sendo menores em pessoas mais velhas (30-34 e $>=35$ anos), $(P=0,00)$, as mulheres apresentaram uma ligeira diminuição no estresse percebido em comparação com os homens $(P=0,04)$ e as pessoas que vivem em áreas rurais apresentam níveis de estresse mais elevados. Discussão: 0 presente estudo revela os efeitos de três meses de reclusão por COVID-19 em uma população de jovens universitários que interromperam sua formação educacional presencial ou virtual, a qual gerou estresse na população de sujeitos do estudo. Conclusão: Evidenciou-se que os homens mais jovens apresentam maiores níveis de estresse em comparação com as mulheres e conforme a idade aumenta, esses níveis diminuem. Morar em áreas rurais pode se tornar um fator de estresse para os alunos.

Palavras chave: Estresse Psicológico; Coronavírus; Estudantes de Ciências da Saúde. 


\section{Introducción}

El término estrés se ha convertido en un vocablo habitual de la sociedad actual, el estrés altera no sólo las respuestas fisiológicas del organismo, sino también los numerosos acontecimientos vitales, las características, se conoce que el estrés es la respuesta psicológica o emocional de un ser humano cuando intenta adaptarse ya sea a presiones internas o externas; inclusive, puede generar estrés hechos positivos que exigen grandes cambios o impliquen algún proceso de adaptación ya sea físico, mental o hasta emocional'.

El contexto universitario es uno de los principales estresores en las personas, debido a que aumentan las exigencias en las académicas y se conoce una relación proporcional el nivel de enseñanza con el nivel de estrés y a medida que aumenta, el estrés de cada persona también va en aumento. Este efecto se conoce como estrés académico, una reacción que activa la parte fisiológica, emocional, cognitiva y conductual que sufren los estudiantes ante ciertos agentes estresores, es decir, estímulos y eventos académicos a los que se encuentran expuestos ${ }^{2}$. Cuando el estrés se encuentra en un nivel adecuado, se tiene una óptima respuesta; pero si el nivel excede las posibilidades de restaurarlo, se convierte en un factor mortal para el rendimiento académico y esta relación se denomina Ley de Yerkes-Dodson ${ }^{3}$.

En los estudiantes del área de la salud este es una problemática como consecuencia de las responsabilidades que se adquieren durante este proceso, el sistema educativo con sus presiones, demandas y disfunciones, pueden llegar a tener efectos negativos sobre la salud, el bienestar y el rendimiento académico de los estudiantes universitarios ${ }^{4}$. Dentro de los estudiantes del área de la salud se puede destacar a los estudiantes de Enfermería, profesión considerada en sí como una profesión estresante, ha sido objeto de investigaciones desde el periodo de formación de futuros profesionales, en las que se ha encontrado que el estrés se relaciona con la ansiedad, pérdida de autoestima y que además interfiere con el aprendizaje y el funcionamiento de los alumnos,

el estrés se relaciona con la ansiedad, pérdida de autoestima y que además interfiere con el aprendizaje y el funcionamiento de los alumnos, desencadenando una posible deserción ${ }^{5}$ desencadenando una posible deserción ${ }^{5}$.

Collazos y Rodríguez ${ }^{6}$ establecieron que la educación de carácter superior representa el punto más alto del estrés académico y por múltiples razones, que van desde las grandes cargas de trabajo hasta la adaptación a los cambios de la vida universitaria, pasando por alteraciones significativas del entorno tanto familiar como social, como un ingreso a la universidad, separación de su familia e inserción al mundo laboral, explicando en una mayor vulnerabilidad de los estudiantes a sufrir periodos de estrés sostenido. Estos agentes originan altos niveles de estrés académico, los cuales serían percibidos con mayor intensidad en estudiantes mujeres que en varones ${ }^{7}$. Si a ello se adicionan las prácticas clínicas que deben desarrollar los estudiantes en los diferentes centros de atención hospitalaria, los niveles de estrés se elevan aún más, debido a la incertidumbre que afrontan ante el surgimiento de una urgencia o algún otro imprevisto y sobre todo al riesgo de causar algún daño al paciente durante la ejecución de algún procedimiento ${ }^{8,9}$.

Al ser este tema de interés para los investigadores como es el caso Montalvo $\mathrm{P}^{10}$, quien evaluó el nivel de estrés aumentados en los estudiantes del área de la salud de Cartagena, reportando que el $88,7 \%$ presentaron la patología anteriormente dicha, reflejándose en síntomas como 
lo son cansancio, ansiedad y cambios en sus patrones de alimentación. De la misma manera, Díaz informó que, en su estudio realizado con estudiantes de Medicina en Cuba, el 60,9\% eran vulnerables a presentar estrés académico; siendo la principal causa generadora de este la sobrecarga académica ${ }^{11}$.

Entre tanto, las principales causas de estrés en los estudiantes del área de la salud y en especial los de Enfermería además de la sobrecarga, son la realización de presentaciones tanto orales como escritas, las altas demandas de trabajo y la realización de las prácticas clínicas que asumen los estudiantes las cuales aumentan de complejidad a medida que avanzan un nivel de formación y en función de los objetivos curriculares que deben alcanzar, según lo informa el estudio realizado por De Dios ${ }^{12}$, por su parte Chávez y Peralta ${ }^{13}$, establecen en su estudio que en cuanto a los síntomas de estrés, se encontró que más de la mitad $(54,8 \%)$ refirieron un nivel moderado, mientras que un $16,7 \%$ señala altos niveles. La presencia de estos síntomas de manera crónica puede afectar severamente la autoestima del estudiante por desgaste emocional. Asimismo, el $78,6 \%$ registraron un nivel medio de estrategias de afrontamiento frente al estrés, mientras que un $14,3 \%$ señala un nivel alto del mismo.

Adicionalmente a estos estresores ya conocidos se suma la presencia de determinantes ambientales que alteran la salud mental de los estudiantes, como es la pandemia mundial del covid- 19, un brote de una nueva neumonía por coronavirus en Wuhan (Hubei, China) que surgió a principios de $2020^{14}$, a raíz de su rápida propagación se ha producido un periodo de confinamiento obligatorio el cual ha traído consigo problemas como estrés, ansiedad y depresión, tanto en el personal médico, como en la población general ${ }^{15}$.

Entre las adaptaciones que se ha producido se encuentra la realización de actividades académicas virtuales, las cuales se constituyen en un reto para las instituciones educativas y especial para los estudiantes del área de la salud quienes deben realizar prácticas clínicas para cumplir con sus actividades de formación, lo que incrementa la ansiedad y el estrés
Entre las adaptaciones que se ha producido se encuentra la realización de actividades académicas virtuales, las cuales se constituyen en un reto para las instituciones educativas y especial para los estudiantes del área de la salud al no poder finalizar sus procesos académicos en especial en las personas que no cuentan con buenos accesos a estas tecnologías ${ }^{2,16}$.

A raíz de esto se propone realizar esta investigación con el fin de conocer el estrés percibido en periodo de confinamiento en estudiantes de Enfermería de la costa caribe de Colombia, documento que puede servir para la creación de estrategias para crear nuevas estrategias académicas para ayudar a la población estudio a reducir los niveles de estrés y contribuir así con la formación integral de los futuros profesionales.

Debido a esto se planteó como objetivo de este estudio determinar el estrés percibido en el periodo de confinamiento por Covid-19 en estudiantes de una universidad de la región Caribe Colombiana en el año 2020. 


\section{Materiales y Métodos}

Estudio de naturaleza cuantitativa, de tipo analítico de corte transversal en el que participaron 398 estudiantes de Enfermería de una Universidad de la costa Caribe Colombiana, fueron incluidos aquellos estudiantes que se encuentran matriculados académica y financieramente, durante el primer periodo de 2020.

Para la recolección de la información se hizo uso de dos instrumentos, el primero es una encuesta elaborada por los autores para evaluar las características sociodemográficas de los estudiantes y el segundo fue la de Estrés percibido 10, creada por Cohen y Williamson en 1988 y validada por Campo A, Oviedo H, Herazo E, en estudiantes de Bucaramanga Colombia, mide la percepción de estrés psicológico y la medida en que las situaciones de la vida cotidiana se aprecian como estresantes, brinda cinco opciones de respuesta:'nunca,' 'casi nunca', 'de vez en cuando,' 'muchas veces' y "siempre', que se clasifican de cero a cuatro. No obstante, los ítems 4, 5, 7 y 8 se califican en forma reversa o invertida, cuenta con una alfa de Cronbach de 0,65.

El análisis de los datos se desarrolló mediante el paquete estadístico SPSS (Statistical Package for Social Studies, versión 22.0). Los resultados de las variables sociodemográficas se presentaron como frecuencias relativas y absolutas por categorías. Adicionalmente se calcularon los promedios y desviaciones estándar (SD) de las puntuaciones obtenidas con la escala de Estrés percibido 10. Se emplearon las pruebas t de Student o ANOVA-one-way para comparaciones entre dos o más medias respectivamente, previa comprobación del supuesto de homogeneidad de varianza mediante el test de Levene. Adicionalmente para los análisis con ANOVA se empleó el test Tukey como prueba Post-Hoc. Los estadísticos se trabajaron con un $95 \%$ de confianza y un $\mathrm{p}$-Valor $<0,05$ se estableció como indicador de significancia estadística para las pruebas aplicadas.

De acuerdo con lo establecido en la resolución No 008430 de 1993 de Colombia y el Código de Helsinki, esta investigación se clasifica como estudio de bajo riesgo para los participantes, dado que hubo una mínima alteración de las variables emocionales y psicológicas. Se contempló el consentimiento informado, la participación voluntaria y el manejo confidencial de los datos. Este proyecto fue aprobado por el comité de investigaciones del programa de Enfermería de la Corporación Universitaria Rafael Núñez mediante en su Acta 002 IP 2020 del 11 mayo del 2020.

\section{Resultados}

\section{Características sociodemográficas de la población de estudio}

En el presente estudio participaron 398 estudiantes de Enfermería de una Universidad de la Costa Caribe de Colombia, donde predominó el sexo femenino con un 92,2\% (367), el rango de edad más frecuente fue de 15 a 19 años con un 37,7\% (150). Se evidenció además que el estrato social al que pertenecían los participantes fue el bajo en el 81,9\% (326), procedieron del área urbana el 74,9\% (298), en cuanto al tipo de familia de los participantes fue la nuclear con un $66,8 \%$ (266) (Tabla 1). 
Tabla 1. Características sociodemográficas y promedio de los puntajes de la escala de estrés percibido $(n=398)$

\begin{tabular}{lccccc}
\hline \multicolumn{1}{c}{ Categoría } & $\mathbf{N}$ & $\%$ & Media & SD & P-Valor \\
\hline Sexo & & & & & 0,04 \\
$\quad$ Femenino & 367 & 92,2 & 18,9 & 5,3 & \\
$\quad$ Masculino & 31 & 7,8 & 18,1 & 3,7 & \\
Edad (años) & & & & & 0,00 \\
15-19 & 150 & 37,7 & 19,9 & 5,6 & \\
$20-24$ & 146 & 36,7 & 18,9 & 4,7 & \\
$25-29$ & 55 & 13,8 & 18,4 & 4,2 & \\
$30-34$ & 22 & 5,5 & 15,4 & 4,9 & \\
$\geq 35$ & 25 & 6,1 & 16,0 & 5,9 & \\
Estrato Social & & & & & \\
Bajo (1 y2) & 326 & 81,9 & 18,9 & 5,2 & \\
Medio (3 y 4) & 72 & 18,1 & 18,3 & 5,4 & \\
Procedencia & & & & & 0,08 \\
Rural & 100 & 25,1 & 19,6 & 4,2 & \\
Urbano & 298 & 74,9 & 18,6 & 5,5 & \\
Tipo de Familia & & & & & 0,13 \\
Nuclear & 266 & 66,8 & 18,6 & 5,0 & \\
Monoparental & 55 & 13,8 & 18,5 & 5,5 & \\
Extensa & 63 & 15,8 & 20,2 & 5,7 & \\
De nuevo tipo & 14 & 3,5 & 18,9 & 4,5 & \\
\hline
\end{tabular}

SD: desviación estándar, $\mathrm{P}=$ valor de $\mathrm{p}$ Test ANOVA.

Fuente: elaboración propia.

\section{Análisis del nivel de estrés percibido según características sociodemográficas $(n=398)$}

En general, en la población de estudio se obtuvo una puntuación promedio de 18,83 5,19 según la escala de estrés percibido, lo que indica que los estudiantes de Enfermería, presentaron niveles de estrés percibido leves. Los valores de las medias (Tabla 1) muestran una aparente tendencia a la disminución en la puntuación del estrés percibido a medida que incrementa el grupo etario. El análisis ANOVA reveló que entre los grupos de edad existen diferencias significativas respecto a las medias del puntaje de estrés. Según la prueba post-Hoc, estas puntuaciones no difieren significativamente entre los grupos de 15-19, 20-24 y 25-29 años. Sin embargo,

La puntuación del estrés percibido a medida que incrementa el grupo etario.

Se puedo observar además que las personas pertenecientes al sexo femenino presentaban una leve disminución del estrés percibido en comparación con los hombres. estos grupos mostraron valores en la puntuación significativamente más altos en comparación los grupos de mayor edad (30-34 y >=35 años), se puedo observar además que las personas pertenecientes al sexo femenino presentaban una leve disminución del estrés percibido en comparación con los hombres. En lo que concierne a los niveles de estrés que presentaron las personas en el área rural presentaron niveles de estrés superiores a los del área urbana con puntaciones de 19,6 y SD 4,2 y de 18,6 SD 5,5 respectivamente. 


\section{Discusión}

El aislamiento social producto de las enfermedades epidémicas, generan un impacto en la salud mental de los individuos expuestos a estas cargas emocionales; algunas de estas alteraciones se relacionan con trastornos mentales como el TEPT, el TDM y los trastornos de ansiedad. En la actualidad, el mundo se encuentra viviendo los estragos de una nueva pandemia denominada COVID-19, un virus procedente de Wuhan-China que accenso a más de 200 países y hoy cobra un número significativo de vidas a nivel mundial. Las decisiones gubernamentales para mitigar los efectos del brote han provocado respuestas emocionales en algunas personas, no obstante, autores estiman que serán muchos más los afectados después de la pandemia ${ }^{15}$.

El presente estudio revela efectos de tres meses de confinamiento por COVID-19 en una población de jóvenes universitarios que interrumpieron su formación educativa de forma presencial a remota, la cual provocó estrés en la población sujeto de estudio. Situación similar se presentó en un grupo de salvadoreños principalmente dependientes, jóvenes estudiantes y mujeres que durante la pandemia por COVID-19 experimentaron estrés, ansiedad y depresión producto de la limitación de la movilidad y el confinamiento domiciliario, así como a la calidad de las relaciones familiares y al nivel de afrontamiento individual ${ }^{16}$.

Aunque el SARS-COV-2 no representa un riesgo para la población joven sana, si impacta en su salud mental, dado que sus efectos provocan mayor carga de estrés en este grupo poblacional, no obstante, a medida que incrementa el grupo etario disminuye la puntuación del estrés percibido. Hallazgos que se asemejan a los resultados de Ozamiz et al, donde se encontraron medias superiores de estrés, ansiedad y depresión en las personas de 18 a 25 años y fue inferior en los mayores de 60. Contexto que pudo beneficiarse del estrés de los jóvenes por adaptarse a estudiar de forma no presencial, pese a las estrategias educativas online que implementaron las instituciones educaciones de educación ${ }^{17}$.

Por otra parte, se logró establecer que las personas del sexo femenino presentaban una leve disminución del estrés percibido en comparación con los hombres. Tal como lo expone Jaimes et $\mathrm{al}^{18}$, se detectó que los mayores niveles de ansiedad se observaron en los hombres. Pero difieren con Valiente y $\mathrm{Cols}^{19}$, en donde las diferencias más significativas en ansiedad fueron en relación con el género femenino, el historial previo de problemas de salud mental, así como el aumento de consumo durante el confinamiento.

En respuesta a los resultados declarados, la universidad como actor básico en la problemática y ente responsable de la sociedad, debe ejercer mayor compromiso e implementar estrategias efectivas e integrales (individuo, familia y sociedad) que le permitan al estudiante afrontar satisfactoriamente las necesidades que sobrevienen de los efectos de la pandemia por COVID-19; estos van desde el suministro de elementos tecnológicos y acceso a internet en especial en los estudiantes que habitan en las áreas rurales donde se cuenta con redes que soporten el acceso a internet para el desarrollo de las actividades académicas, como la promoción del autocuidado para la protección, prevención y mantenimiento de salud mental. Además, de aquellas intervenciones que la Instituciones de Educación Superior vienen realizando, como el apoyo académico, psicológicos y trabajar en las competencias necesarias de los jóvenes para el manejo de las redes sociales y el estrés ${ }^{20}$. 
Este estudio limita los efectos del COVID-19 al periodo de confinamiento, no obstante, se debe prestar atentación a las manifestaciones de los grupos más vulnerables de presentar síntomas de estrés postraumático, para intervenir de forma oportuna; algunos de estos indicadores predictores son: ser joven, mujer, convivir con menores, consumir sustancias durante el confinamiento ${ }^{21,22}$, personas con alto riesgo de infección o que hayan tenido contacto cercano con pacientes $\underline{23}$.

Las evidencias anteriores, muestra una problemática que constituye un foco de atención importante teniendo en cuenta que la población más afectada por este flagelo correspondió al grupo etario más joven, los cuales además de vivir la transición de la educación básica, lidian con los cambios propios del ciclo vital. A ello, se le suma las consecuencias del estrés que pueden conducir hasta la deserción universitaria.

Finalmente, estos aspectos proveen una base para comprender algunos efectos del confinamiento producto del brote por Covid 19 en la salud mental de jóvenes universitarios, permitiendo una mejor orientación de las intervenciones en salud y un

Estos aspectos proveen una base para comprender algunos efectos del confinamiento producto del brote por Covid 19 en la salud mental de jóvenes universitarios mejor abordaje de las práctica de promoción, protección y mantenimiento de la salud mental propuestas por las instituciones de educación superior frente a contingencia de origen sanitario que sin duda impacta directamente en desarrollo integro de la formación educativa.

\section{Conclusiones}

Los estudiantes de Enfermería de Universidad de la costa Caribe Colombiana mostraron niveles leves de estrés percibido durante el periodo de confinamiento, encontrándose diferencias significativas en personas con menor edad y quienes tienen un rango de edad de 30 a 35 años, además fue más común encontrarlas en hombres y personas que habitan en áreas rurales donde el acceso a internet es difícil y no pueden desarrollar sus actividades académicas.

Conflicto de interés: Los autores declaran que no hubo conflicto de intereses durante esta investigación. El estudio no tenía vínculos personales, financieros y políticos con las entidades o individuos encuestados.

Financiación: Ninguno

\section{Referencias}

1. Espinoza A, Pernas I, González R. Consideraciones teórico-metodológicas y prácticas acerca del estrés. Humanidades Médicas. 2018;8(3),697-717.

2. Barraza A. El estrés académico de los alumnos de Educación Media Superior, Hermosillo. Memoria electrónica del VIII Congreso Nacional de Investigación Educativa, 2015. Disponible en: https://dialnet.unirioja.es/descarga/articulo/2358918.pdf

3. Barraza A. Características del estrés académico en los alumnos de educación media superior. Investigación Educativa Duranguense. 2015;(4):15-20.

4. Jerez M, Oyarzo C. Estrés académico en estudiantes del Departamento de Salud de la Universidad de Los Lagos Osorno. Rev. chil. neuro-psiquiatr. 2015;53(3):149-157.

https://doi.org/10.4067/S0717-92272015000300002 
5. Galvin J, Suominen E, Morgan C, O'Connell EJ, Smith AP. Mental health nursing students' experiences of stress during training: mathematic analysis of qualitative interviews $J$ PsychiatrMent HealthNurs. 2015;22(10):773-83. https://doi.org/10.1111/jpm.12273

6. Collazo C, Rodríguez Y. El estrés académico: una revisión crítica del concepto desde las ciencias de la educación. Revista Electrónica de Psicología Iztacala. 2011;14(2):1:1870-8420

7. Cabanach R, Souto-Gestal A, Franco V. Escala de Estresores Académicos para la evaluación de los estresores académicos en estudiantes universitarios. Revista iberoamericana de psicología y salud. 2016;7(2):41-50. https://doi.org/10.1016/j.rips.2016.05.001

8. Oro P, Esquerda M, Viñas, Yuguero O, Pifarre J. Síntomas psicopatológicos, estrés y burnout en estudiantes de medicina. Educación Médica. 2019;20(S1):42-48.

https://doi.org/10.1016/j.edumed.2017.09.003

9. Lópezl, SánchezV.Percepción del estrés en estudiantes de enfermería en las prácticas clínicas. Enfermería Clínica. 2015;15(6):307-13. https://doi.org/10.1016/S1130-8621(05)71136-0

10.Montalvo A, Blanco K, Cantillo N, Castro Y, Downs A, Romero E. Estrés académico en los estudiantes del área de la salud en una universidad pública, Cartagena-Colombia. Revista Ciencias Biomédicas. 2015;6(2):309-18. https://doi.org/10.32997/rcb-2015-2959

11.Díaz Y. Estrés académico y afrontamiento en estudiantes de Medicina. Hum Méd. 2010;10(1):1727-8120

12.De Dios M, Varela I, Braschi L, Sánchez E. Estrés en estudiantes de enfermería. Educ Med Super. 2017;31(3):110-123.

13.Estrés académico y autoestima en estudiantes de enfermería, Arequipa-Perú. Chávez J, Peralta R. Revista de Ciencias Sociales (RCS). XXV. 1.2019. Disponible en: https://produccioncientificaluz.org/index.php/rcs/article/view/29629

14.Chen Q, Liang M, Li Y, Guo J, Fei D, Wang L, et al. Mental health care for medical staff in China during the COVID-19 outbreak. Lancet Psychiatry 2020;7:e15-6. https://doi.org/10.1016/S2215-0366(20)30078-X

15.Ramírez J, Castro Q, Lerma C, Yela F, Escobar F. Consecuencias de la pandemia covid 19 en la salud mental asociadas al aislamiento social. 2020. Disponible:

https://preprints.scielo.org/index.php/scielo/preprint/view/303/358

16.Orellana C, Orellana L. Predictores de síntomas emocionales durantela cuarentena domiciliar por pandemia de COVID-19 en EI Salvador. Actualidades en Psicología, 2020;34(128):103-120. https://doi.org/10.15517/ap.v34i128.41431

17.Ozamiz N, Dosil-Santamaria M, Picaza-Gorrochategui M, Idoiaga-Mondragon N. Niveles de estrés, ansiedad y depresión en la primera fase del brote del COVID-19 en una muestra recogida en el norte de España. Cadernos de Saúde Pública, 2020;36:e00054020. https://doi.org/10.1590/0102-311x00054020

18.Jaimes N, Espinosa A, Hernández M, Zeus O. Impacto psicológico en estudiantes universitarios mexicanos por confinamiento durante la pandemia por Covid-19. 2020. https://doi.org/10.1590/SciELOPreprints.756

19.Valiente C, Vázquez C, Peinado V, Contreras A, Trucharte A, Bentall R, Martínez A. VIDACOVID-19 Estudio nacional representativo de las respuestas de los ciudadanos de España ante la crisis de Covid-19: respuestas psicológicas. 2020. Disponibles: https://tribuna.ucm.es/revcul/tribunacomplutense/doc24997.pdf

20.Huarcaya J. Consideraciones sobre la salud mental en la pandemia de COVID-19. Revista Peruana de Medicina Experimental y Salud Pública, 2020;37(2). https://doi.org/10.17843/rpmesp.2020.372.5419

21.Sun L, Sun Z, Wu L, Zhu Z, Zhang F, Shang Z, Liu N. Prevalence and risk factors of acute posttraumatic stress symptoms during the COVID-19 outbreak in Wuhan, China. MedRxiv, 2020. https://doi.org/10.1101/2020.03.06.20032425 
22.Liu S, Yang L, Zhang C, Xiang Y-T, Liu Z, Hu S, et al. Online mental health services in China during the COVID-19 outbreak. Lancet Psychiatry 2020;7:e17-8. https://doi.org/10.1016/S2215-0366(20)30077-8

23.Departamento de Salud, Gobierno Vasco. Nuevo coronavirus (COVID-19). Disponible en: http://www.euskadi.eus/nuevo-coronavirus-covid-19/ 